\title{
Cytokeratin 13, Cytokeratin 17, and Ki-67 Expression in Human Acquired Cholesteatoma and Their Correlation With Its Destructive Capacity
}

\author{
Mahmood A. Hamed ${ }^{1,2} \cdot$ Seiichi Nakata ${ }^{1} \cdot$ Kazuya Shiogama $^{3} \cdot$ Kenji Suzuki $^{1,4} \cdot$ Ramadan H. Sayed $^{2} \cdot$ Yoichi Nishimura $^{1}$ \\ Noboru Iwata ${ }^{1} \cdot$ Kouhei Sakurai $^{5} \cdot$ Badawy S. Badawy $^{2} \cdot$ Ken-ichi Inada $^{5} \cdot$ Hayato Tsuge $^{6} \cdot$ Yutaka Tsutsumi $^{3}$ \\ ${ }^{I}$ Department of Otorhinolaryngology, Banbuntane-Hotokukai Hospital, Fujita Health University School of Medicine, Nagoya, Japan; \\ ${ }^{2}$ Department of Otorhinolaryngology, Sohag Faculty of Medicine, Sohag University, Sohag, Egypt; ${ }^{3}$ Department of Pathology, Fujita Health \\ University School of Medicine, Toyoake; ${ }^{4}$ Department of Otorhinolaryngology, Yonaha General Hospital, Kuwana; ${ }^{5}$ Department of Pathology, \\ Banbuntane-Hotokukai Hospital, Fujita Health University School of Medicine, Nagoya; ${ }^{6}$ Department of Otorhinolaryngology, Red Cross Nagoya \\ Daiichi Hospital, Nagoya, Japan
}

Objectives. Cholesteatoma is a nonneoplastic destructive lesion of the temporal bone with debated pathogenesis and bone resorptive mechanism. Both molecular and cellular events chiefly master its activity. Continued research is necessary to clarify factors related to its aggressiveness. We aimed to investigate the expression of Ki-67, cytokeratin 13 (CK13) and cytokeratin 17 (CK17) in acquired nonrecurrent human cholesteatoma and correlate them with its bone destructive capacity.

Methods. A prospective quantitative immunohistochemical study was carried out using fresh acquired cholesteatoma tissues $(n=19)$, collected during cholesteatoma surgery. Deep meatal skin tissues from the same patients were used as control $(\mathrm{n}=8)$. Cholesteatoma patients were divided into 2 groups and compared (invasive and noninvasive) according to a grading score for bone resorption based upon clinical, radiologic and intraoperative findings. To our knowledge, the role of CK17 in cholesteatoma aggressiveness was first investigated in this paper.

Results. Both Ki-67 and CK17 were significantly overexpressed in cholesteatoma than control tissues $(P<0.001$ for both Ki-67 and CK17). In addition, Ki-67 and CK17 were significantly higher in the invasive group than noninvasive group of cholesteatoma $(P=0.029, P=0.033$, respectively). Furthermore, Ki-67 and CK17 showed a moderate positive correlation with bone erosion scores $(r=0.547, P=0.015$ and $r=0.588, P=0.008$, respectively). In terms of CK13, no significant difference was found between cholesteatoma and skin $(P=0.766)$.

Conclusion. Both Ki-67 and CK17 were overexpressed in cholesteatoma tissue and positively correlated with bone resorption activity. The concept that Ki-67 can be a predictor for aggressiveness of cholesteatoma was supported. In addition, this is the first study demonstrating CK17 as a favoring marker in the aggressiveness of acquired cholesteatoma.

Keywords. Cholesteatoma; Middle Ear; Bone Resorption; Ki-67 Antigen; Cytokeratin 17

\footnotetext{
- Received August 31, 2016

Revised December 10, 2016

Accepted December 14, 2016

- Corresponding author: Mahmood A. Hamed

Department of Otorhinolaryngology, Banbuntane-Hotokukai Hospital, Fujita Health University School of Medicine, 3-6-10 Otobashi, Nakagawaku, Nagoya 454-8509, Japan

Tel: +81-52-323-5647, Fax: +81-52-331-6843

E-mail: mahmoodhamed8@gmail.com
}

\section{INTRODUCTION}

Cholesteatoma is a proliferative lesion of the temporal bone which exhibits varied growth characteristics compared with the normal epidermis. Its pathogenesis as well as the mechanism of bone resorption have yet to be elucidated [1,2]. Different molecules were proven to have a significant role in the pathogenesis as well as in bone resorption activity of cholesteatoma, includ-

Copyright @ 2017 by Korean Society of Otorhinolaryngology-Head and Neck Surgery.

This is an open-access article distributed under the terms of the Creative Commons Attribution Non-Commercial License (http://creativecommons.org/licenses/by-nc/4.0)

which permits unrestricted non-commercial use, distribution, and reproduction in any medium, provided the original work is properly cited. 
ing cytokines, proliferation markers, enzymes and lipopolysaccharides [3-7].

Cholesteatoma fundamentally belongs to hyperproliferative disorders [6]. However, whether or not the hyperproliferative capacity is correlated with its aggressive pattern remains controversial $[7,8]$. Several proliferative markers were found to be overexpressed in cholesteatoma tissues, such as Ki-67 (MIB-1), telomerase, transforming growth factor- $\alpha$, keratinocyte growth factor, and amphiregulin [7,9-12]. Additionally, some molecules were found to be related to the bone resorption capacity of cholesteatoma and therefore, could predict its behavior. Of these molecules are tumor necrosis factor- $\alpha$, interleukin- $1 \alpha$, epidermal growth factor [13], matrix metaloproteinase-9, tenascin [14] and bone morphogenic proteins [15]. This knowledge has improved our understanding of this serious disease and also paved the way for alternative therapeutic strategies other than surgical intervention [16].

In this article, we conducted an immunohistochemical study to investigate the expression profile of Ki-67, a cell proliferation marker, cytokeratin 13 (CK13) and CK17, markers of keratinocyte differentiation, in cholesteatoma tissues and detect their role in bone destruction. Acquired nonrecurrent cholesteatoma tissues were collected during cholesteatoma surgery. Control skin tissues were sampled from the bony portion of the external auditory canal. The bone resorption activity of cholesteatoma was determined by a grading score based upon the number of eroded bones seen in radiological and intraoperative findings.

To the best of our knowledge, the expression and role of CK17 in human cholesteatoma tissues and meatal skin was seldom seen in previous reports $[17,18]$.

\section{MATERIALS AND METHODS}

\section{Samples and grading score}

A prospective experimental study was employed. The present study complied with the ethical standards of the relevant regional and institutional guidelines on human studies and with Declaration of Helsinki. All patients included in this study gave a written informed consent for their participation. Cholesteato-

\section{H I I G H L I I G H T T S}

- Bone resorption in human acquired cholesteatoma is still a confusing mystery.

- The concept that Ki-67 could predict cholesteatoma aggressiveness was supported.

- Cytokeratin 17 could be considered as a favoring marker for cholesteatoma invasiveness.

- This knowledge is necessary for proper timing of surgery and preventing bad outcomes.
Table 1. Grading score of bone erosion of cholesteatoma used in our study (newly developed)

\begin{tabular}{ll}
\hline Bone & \multicolumn{1}{c}{ Grading score } \\
\hline Scutum & Intact $=0$ \\
& Eroded $=1$ \\
Auditory ossicles & Intact $=0$ \\
& One ossicle eroded $=1$ \\
& $\geq 2$ Ossicles $=2$ \\
Intact $=0$
\end{tabular}

Complications were included as the highest grade for each bone specifically.

ICC, intracranial complications; PCW, posterior canal wall.

ma tissues were collected during tympanomastoid surgery for patients diagnosed as having acquired nonrecurrent cholesteatoma as the study group ( $\mathrm{n}=19)$, and operated in our institute between August 2014 and April 2016. Congenital and recurrent cases were excluded. Control tissues were collected from the skin of the deep part of the external auditory canal $(n=8)$. All the control skin tissues had their corresponding cholesteatoma tissues. The patients consisted of 11 males and 8 females, with a mean age of 54 years (range, 16 to 80 years). The patients were classified into 2 groups, according to the bone resorption activity of cholesteatoma based on clinical, radiological and intraoperative findings. Grading of invasiveness was done by counting the number of eroded bones and the presence of one or more complication to be considered as the highest grade for each bone specifically (Table 1). Concerning the facial canal dehiscence, only cases with direct contact with cholesteatoma debris were scored. The final grading score was as follows: noninvasive group (no or mild bone erosion), score $0-3$; invasive group (severe or advanced bone erosion), score $\geq 4$ (Table 1 ).

Specimens were immediately fixed in $10 \%$ neutral-buffered formalin for a maximum of 24 hours, then formalin fixed paraffin embedded (FFPE) blocks were prepared and kept for histopathological and immunohistochemical examinations. 


\section{Histopathology and immunostaining}

FFPE tissues were cut at $3 \mu \mathrm{m}$ thickness and mounted. First, hematoxylin and eosin stained slides were evaluated, and tissues that included the full thickness of cholesteatoma with matrix (keratinized squamous epithelium) and perimatrix (subepithelial connective tissue) were chosen for further immunostaining. We started with 28 cholesteatomas and 13 skin tissues. Nine cholesteatomas and 5 skin tissues were excluded. The study was thus finally performed using 19 cholesteatoma samples and 8 skin samples.

To block endogenous peroxidase activity, tissue sections were soaked in methanol containing $0.3 \%$ hydrogen peroxide for 30 minutes at room temperature. Heat-induced epitope retrieval was then performed using a pressure pan for 10 minutes. A preliminary study found that $1 \mathrm{mM}$ ethylene diamine tetraacetic acid solution, $\mathrm{pH} 8.0$ was the optimal soaking heating solution for demonstrating CK13, CK17, and Ki-67. Afterwards, the sections were left to cool at room temperature for 30 minutes, then incubated with primary mouse monoclonal antibodies for CK13 (dilution 1:500; Novocastra, Newcastle, UK; clone KS-1A3), CK17 (dilution 1:400; Dako, Kyoto, Japan; clone E3) and Ki-67 (dilution 1:100; Dako; clone MIB1) overnight at room temperature. Sections were then incubated with the secondary amino acid polymer reagent, Histofine Simple Stain MAX-PO (Nichirei Biosciences, Tokyo, Japan) for 30 minutes at room temperature. Reaction products were visualized by incubating the sections in a 3,3'-diaminobenzidine solution containing $0.006 \%$ hydrogen peroxide for 5 minutes. Finally, the nuclei were lightly counterstained with Mayer's hematoxylin.

\section{Evaluation of immunostaining}

Evaluation was done by 2 independent observers, one of them is a pathologist and the other is the researcher himself. The cytoplasmic staining for CK13 and CK17 and nuclear labeling for Ki67 were considered positive. The percentage of the positive cells was determined in 3 different areas at 200 magnification under a light microscope. Namely, the percentage of positive cells in the entire keratinocyte layer was counted. Patients' demographics, scores and the expression profiles were described in Table 2.

\section{Statistical analysis}

For descriptive purposes, we evaluated both mean \pm 1 standard error of the mean (SE) and median/interquartile range (IQR) according to normality of data distributions. Measurable variables were compared by using Student $t$-test for independent samples for CK17 and Ki-67, and by using Mann-Whitney U-test for CK13. Spearman rho correlation was employed for correlating the expression of Ki-67 and CK17 with the grading score of bone erosion. Wilcoxon signed-rank test was used to compare CK13 and CK17 expressions in the paired skin and cholesteatoma tissues. All the statistical analyses were done using an SPSS ver. 16.0 (SPSS Inc., Chicago, IL, USA) in 2007. $P$-values less than
Table 2. Patients' demographics and expression profiles of Ki-67, cytokeratin (CK) 17, and CK13 in cholesteatoma tissues

\begin{tabular}{lccccc}
\hline Patient & $\begin{array}{c}\text { Sex/age } \\
(\mathrm{yr})\end{array}$ & $\begin{array}{c}\mathrm{GSB}^{*} \\
(\text { score })\end{array}$ & $\begin{array}{c}\text { Ki-67 } \\
(\%)\end{array}$ & $\begin{array}{c}\text { CK17 } \\
(\%)\end{array}$ & $\begin{array}{c}\text { CK13 } \\
(\%)\end{array}$ \\
\hline$\# 1$ & $\mathrm{M} / 48$ & 6 & 48 & 82 & 43.5 \\
$\# 2$ & $\mathrm{M} / 69$ & 4 & 41 & 74.2 & 12 \\
$\# 3$ & $\mathrm{M} / 80$ & 7 & 36 & 71.5 & 42.5 \\
$\# 4$ & $\mathrm{M} / 35$ & 8 & 46.7 & 85 & 52 \\
$\# 5$ & $\mathrm{M} / 51$ & 5 & 43.7 & 85.5 & 46.5 \\
$\# 6$ & $\mathrm{M} / 54$ & 5 & 41.3 & 64.5 & 68 \\
$\# 7$ & $\mathrm{M} / 64$ & 4 & 50 & 84 & 51 \\
$\# 8$ & $\mathrm{~F} / 78$ & 4 & 33 & 83 & 57 \\
$\# 9$ & $\mathrm{~F} / 67$ & 4 & 44 & 61 & 45 \\
$\# 10$ & $\mathrm{~F} / 75$ & 4 & 29 & 75 & 50 \\
$\# 11$ & $\mathrm{~F} / 42$ & 2 & 34 & 59.5 & 77 \\
$\# 12$ & $\mathrm{~F} / 75$ & 3 & 37 & 71 & 69 \\
$\# 13$ & $\mathrm{~F} / 48$ & 3 & 51 & 69.5 & 0 \\
$\# 14$ & $\mathrm{~F} / 16$ & 1 & 37 & 46 & 66 \\
$\# 15$ & $\mathrm{M} / 16$ & 3 & 28.5 & 68 & 0 \\
$\# 16$ & $\mathrm{~F} / 62$ & 1 & 29 & 65 & 13 \\
$\# 17$ & $\mathrm{M} / 33$ & 3 & 30.6 & 82.5 & 49 \\
$\# 18$ & $\mathrm{M} / 18$ & 2 & 26.6 & 60 & 55 \\
$\# 19$ & $\mathrm{M} / 67$ & 2 & 24.6 & 75 & 39 \\
\hline
\end{tabular}

${ }^{*}$ Grading score of bone erosion of cholesteatoma (0-15).

0.05 were considered significant with a confidence interval $95 \%$.

\section{RESULTS}

\section{Cholesteatoma lesions}

Cholesteatoma involved the right ear in 7 patients (37\%), left ear in 7 patients (37\%) and bilateral in the remaining 5 patients (26\%). Intact canal wall mastoidectomy was performed in 7 patients $(37 \%)$ and canal wall down was performed in 12 patients (63\%). According to the grades of bone erosion, cholesteatoma was categorized into two groups: invasive group $(n=10)$ and noninvasive group $(n=9)$. In our patients, the minimal score was " 1 " while the maximum being " 8 " (Table 2).

\section{Expression of Ki-67, CK17, and CK13}

The difference in the labeling index of Ki-67 in cholesteatoma $(\mathrm{n}=19)$ and the meatal skin $(\mathrm{n}=8)$ was highly significant $(37.44 \% \pm 1.9 \%$ vs. $20.96 \% \pm 1.2 \%, P<0.001)$. Significant difference was also found between the invasive group and noninvasive group $(41.27 \% \pm 2.13 \%$ vs. $33.2 \% \pm 2.6 \%, P=0.029)$ (Fig. 1). In the skin tissues, Ki-67 expression was mainly seen in the nuclei of single-layered parabasal keratinocytes, while in cholesteatoma, the labeled parabasal layer was thickened, with increased labeling in the basal cells. Some columnar cells and stromal cells in the underlying connective tissue were also labeled for Ki-67. Additionally, a moderately significant positive correlation was observed between Ki-67 expression and the 

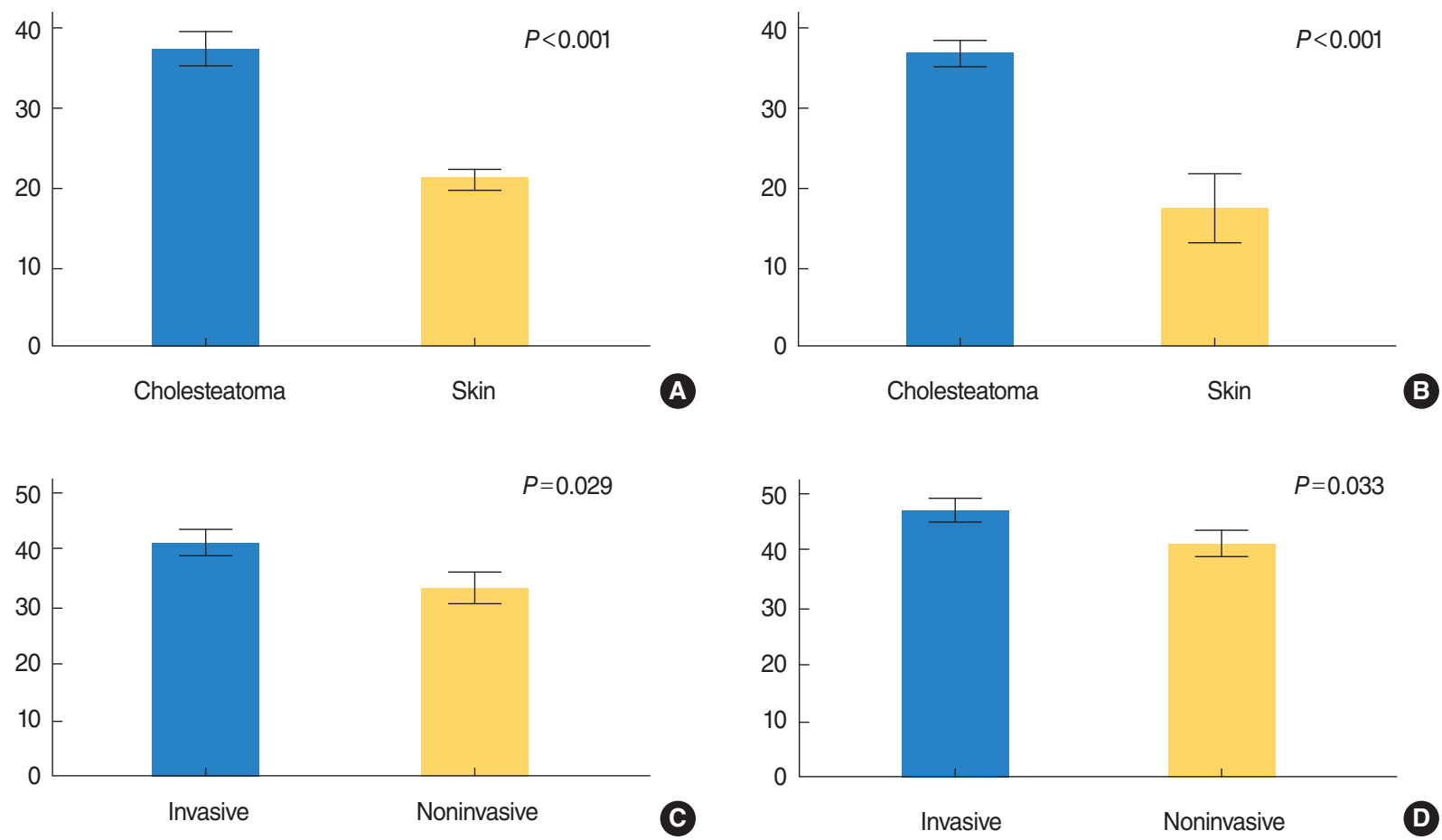

Fig. 1. (A, B) Expression of Ki-67 and cytokeratin 17 (CK17) in cholesteatoma versus skin (tissue group, mean \pm SE) and (C, D) in invasive versus non-invasive cholesteatoma (cholesteatoma group, mean $\pm \mathrm{SE}$ ). Note overexpression of both Ki-67 and CK17 in cholesteatoma compared to skin tissues $(P<0.001)$, and in invasive group than noninvasive one $(P<0.05)$. Independent $t$-test. SE, standard error of the mean.
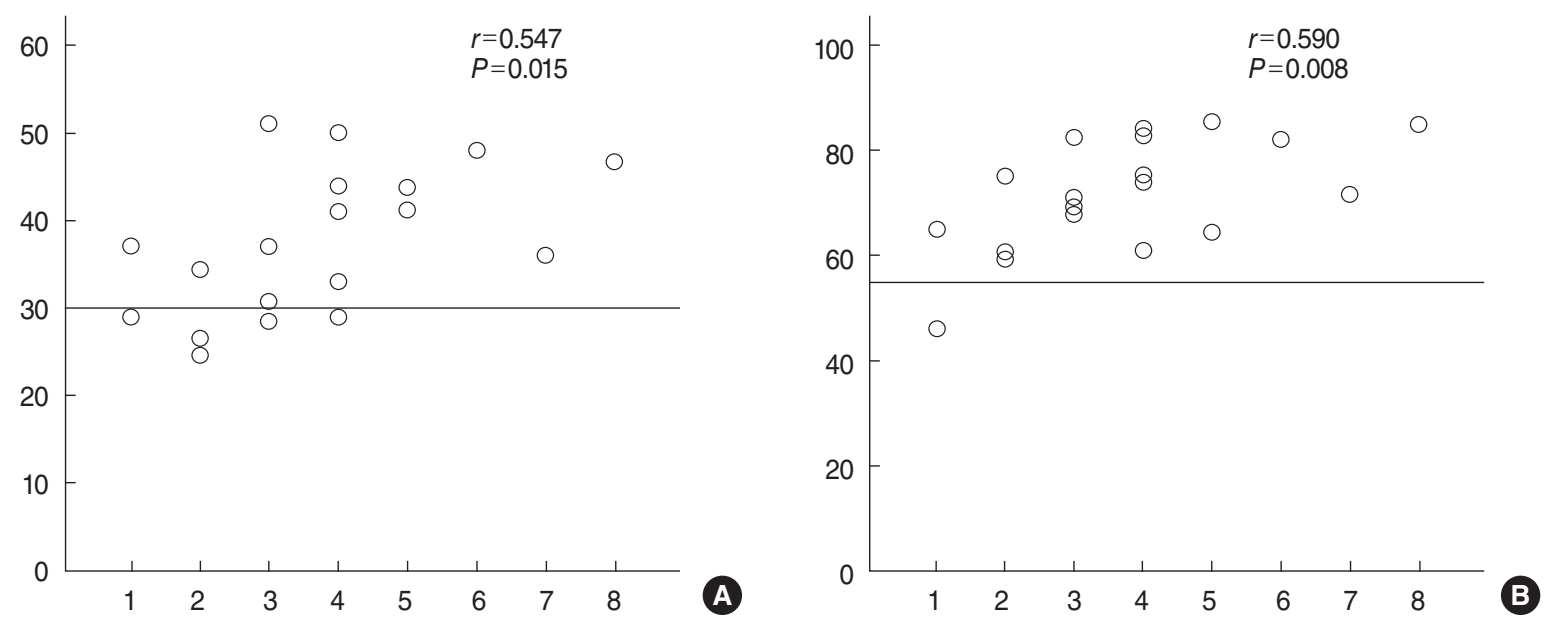

Fig. 2. Spearman correlation of (A) Ki-67 and (B) cytokeratin 17 (CK 17) with scores of bone erosion. Moderate positive correlations were observed between Ki-67 $(r=0.547, P<0.05)$ and CK17 $(r=0.588, P<0.01)$ and grading score used in our study.

grades for bone erosion $(r=0.547, P=0.015)$ (Fig. 2$)$.

Regarding CK17, we found a highly significant difference in its mean expression between cholesteatoma and the meatal skin $(71.71 \% \pm 2.45 \%$ vs. $34.4 \% \pm 8.37 \%, P<0.001)$. The expression of CK17 was significantly increased in invasive cholesteatoma compared with the noninvasive group $(76.57 \% \pm 2.7 \%$ vs. $66.32 \% \pm 3.4 \%, P=0.033$ ) (Fig. 1). The basal and parabasal layers were negative for CK17. It was expressed mainly in the su- prabasal layers, but the expression was consistent in cholesteatoma lesions. Middle ear columnar cells were also positive for CK17. Finally, a moderately significant positive correlation was seen between CK17 expression and the grade of bone erosion $(r=0.588, P=0.008)$ (Fig. 2).

This means that both Ki-67 and CK17 were overexpressed in cholesteatoma, and the expression was activated in the invasive cholesteatoma (Fig. 1). Representative immunostained features 

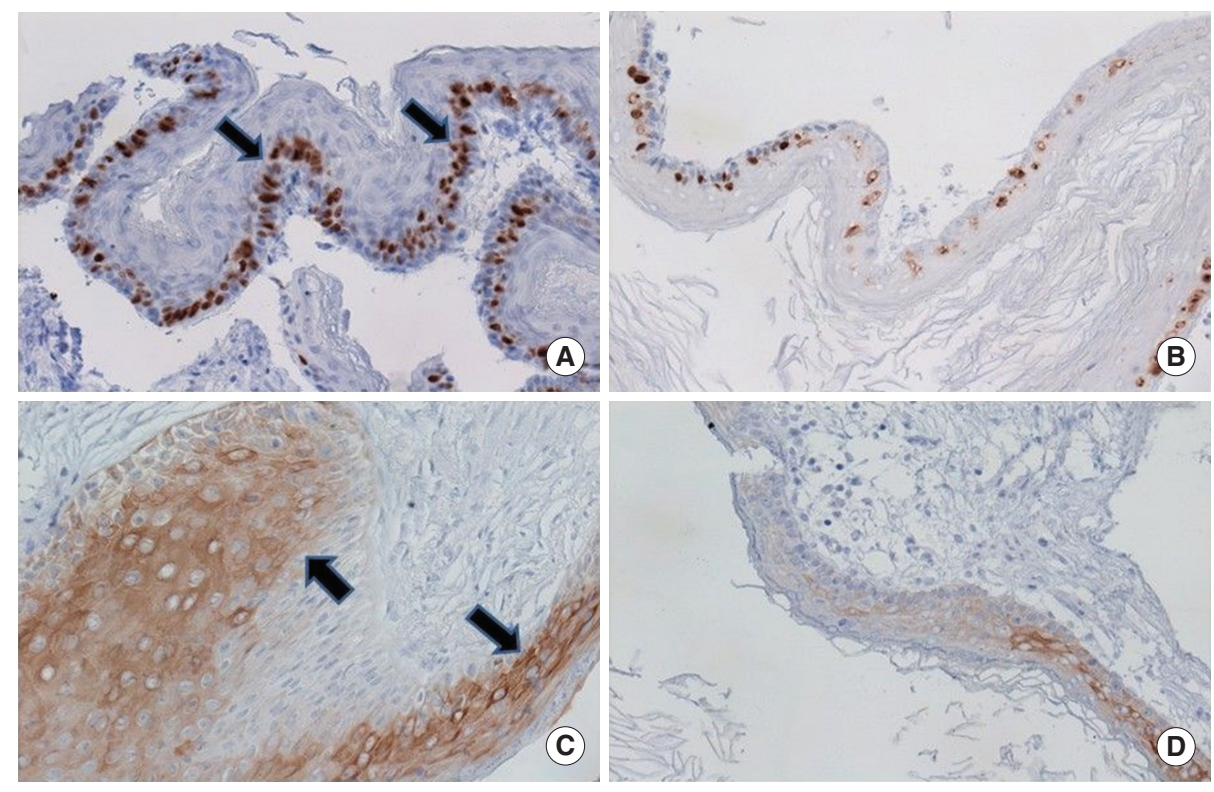

Fig. 3. Expression of (A) Ki-67 in invasive, (B) Ki-67 in noninvasive, (C) cytokeratin 17 (CK17) in invasive, and (D) CK17 in noninvasive cholesteatoma. Overexpression of $\mathrm{Ki}-67$ is observed in the invasive group as compared to the noninvasive one (arrows). The same is true for CK17 (arrows) (Mayer's hematoxylin, ×200).
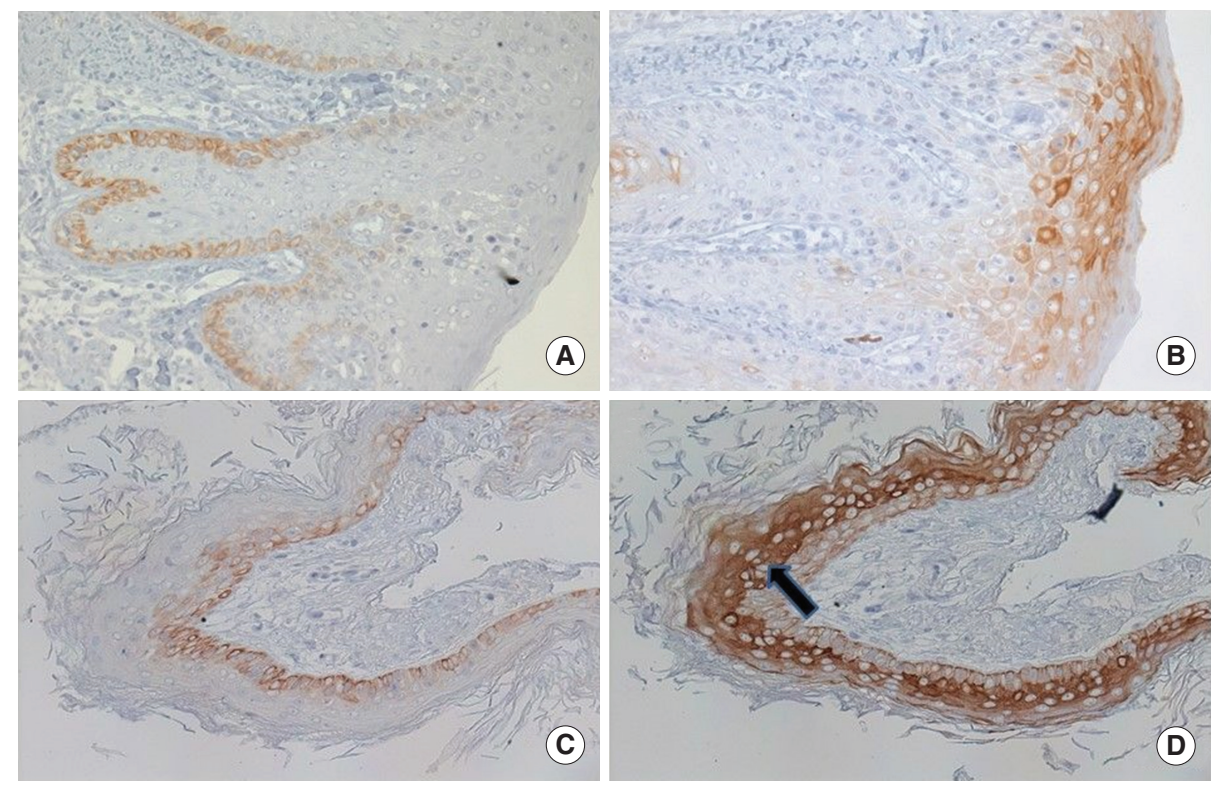

Fig. 4. Expression of (A) cytokeratin 13 (CK13) and (B) CK17 in skin, and (C) CK13 and (D) CK17 in cholesteatoma tissues. Expression of CK17 was much increased in cholesteatoma (arrows) compared to the skin but CK13 did not show much difference in both skin and cholesteatoma tissues (Mayer's hematoxylin, ×200).

are illustrated in (Fig. 3).

Regarding CK13, we did not find significant difference between cholesteatoma and the meatal skin: $49.5 \%$ (17.6) versus $46.7 \%$ (11.1) respectively, $P=0.750$ and no difference between invasive and noninvasive groups $(P>0.05)$. The expression pattern of CK13 was similar in both cholesteatoma and the meatal skin. It was mainly confined to the basal layer and parabasal staining was also occasionally found. All the control skin tissues expressed CK13, while 2 cholesteatoma samples were negative. Columnar cells embedded in the stroma were occasionally positive. There was no significant difference in the expression of CK13 and CK17 in the control skin tissues $(P=0.313)$ but in cholesteatoma, CK17 was highly increased compared with CK13 $(P<0.001)$ (Fig. 4). 
No significant correlation was found between Ki-67 and CK17 $(r=0.251, P=0.300)$. Additionally, no significant correlation between the age of the patients at the time of surgery and our grading score $(r=0.338, P=0.158)$.

\section{DISCUSSION}

\section{Ki-67 labeling index of cholesteatoma and scoring of bone re- sorption}

Ki-67 nuclear antigen identified by MIB-1 monoclonal antibody is known to be detected in proliferative cells especially in the late G1, S, G2, and M phases. It has been widely used as a trusted proliferative marker in tumors as well as nonneoplastic proliferative disorders, including cholesteatoma $[7,19,20]$.

Many previous reports found that Ki-67 was overexpressed in the cholesteatoma epithelium compared to the normal retroauricular and meatal skin [8,19-22]. In contrast to these results, Kuczkowski et al. [23] failed to find any significant difference in Ki-67 expression between 51 cholesteatomas and 6 meatal skin tissues.

However, there was controversy about the use of Ki-67 nuclear antigen as a predictor of cholesteatoma aggressiveness. Mallet et al. [7] correlated Ki-67 expression to the severity of bone destruction. They defined bone erosion as "moderate" when one ossicle was affected and "severe" when more ossicles were eroded and/or nonossicular temporal bone structures of the middle and inner ear were affected. They concluded that cholesteatomas with severe ossicular destruction expressed Ki-67 in an active form (basal cell nuclei were continuously stained), whereas those with less ossicular destruction expressed Ki-67 in an inactive form (basal cell nuclei were stained in an interrupted manner) [7]. Furthermore, Juhasz et al. [14] documented that "destructive" cholesteatomas with evidence of bone resorption had higher Ki-67 leveling than "nondestructive" ones that were completely free of bone resorption. In contrast, Aslier et al. [24], comparing between pediatric and adult cholesteatomas, did not find any correlation between Ki-67 and bone erosion scores of cholesteatoma, by employing a new scoring system for bone erosion graded as " 1 " for incomplete ossicular destruction, and "2" referred to complete ossicular chain destruction but graded " 2 " only in case of destruction of the facial canal, tegmen and otic capsule as partial destruction of these structures could not be elicited. Finally, in an animal study of dogs, Banco et al. [8] stated that the Ki-67 labeling index was higher in cholesteatoma than the meatal skin, but could not be used as a predictor for aggressiveness. Therefore, this subject has yet to be clarified.

The lack of a standardized method to specify bone erosion and to distinguish invasive cholesteatomas from noninvasive ones made it a hard and confusing issue when interpreting and comparing the results [24]. We suggested an easy, strictly objective, broad and applicable scoring system by counting the num- ber of eroded bones seen radiologically and confirmed intraoperatively, putting in mind complicated cases. According to our scoring, grades 0-3 meant that cholesteatoma induced destruction was restricted only to the lateral attic wall (scutum) and ossicular chain (noninvasive group) while grades 4 and more meant that cholesteatoma extended beyond the ossicular chain and tympanic cavity to the mastoid region and other nonossicular temporal bone structures as well (invasive group). Complications were scored as the highest grade for each related bone specifically (Table 1 ).

Concerning the facial canal dehiscence, only cases with direct contact with cholesteatoma debris were scored [24]. Being simple, applicable and more adherent to the point, we see that our grading system was more advantageous than those prescribed in the previously mentioned reports. Nevertheless, we see that there is an indigence to conduct universally approved guidelines to objectively measure cholesteatoma aggressiveness. We confirmed that cholesteatoma expressed higher Ki-67 levels than the meatal skin (the mean ratio was nearly doubled in cholesteatoma, $P<0.001$ ), and the results were compatible with the previous studies [19-21]. Additionally, both higher labeling in the invasive group and moderately positive correlation with the grading score of bone erosion (Table 2) suggest an important role of Ki-67 in bone destruction ( $P=0.029, P=0.015$, respectively). We provided supporting evidence that the proliferative capacity of cholesteatoma as determined by Ki-67 immunostaining significantly correlated with its aggressive clinical behavior.

\section{Expression of CK13 and CK17}

Cytokeratins, a set of intermediate filament proteins of mammalian cells, have been widely used as epithelial markers. The expression patterns of the cytokeratins differ according to the anatomical location, state and stage of differentiation $[25,26]$. Especially, higher molecular weight cytokeratins such as CK13 and CK17 can be immunohistochemical markers of squamous hyperproliferative disorders, including cholesteatoma.

To our knowledge, CK17 expression in cholesteatoma and meatal skin was scarcely discussed in previous reports $[17,18]$. Furthermore, none of these studies correlated CK17 leveling to the degree of cholesteatoma invasiveness. Vennix et al. [17] suggested a striking feature for the deep meatal skin in harboring a special group of hyperproliferative cytokeratins including CK6, CK16, and CK17. These cytokeratins were not normally represented except in injured epidermis as well as in skin diseases. Acquired cholesteatoma was strongly similar to the deep meatal tissues favoring its hyperproliferative nature especially in its "advancing front" [18].

In our study, we investigated the expression of CK13 and CK17 in cholesteatoma and the meatal skin tissue. A pair of CK13 and CK17 has often been used as potential markers for oral dysplasia, oral squamous cell carcinoma (OSCC) and other nonneoplastic proliferative skin lesions such as psoriasis [27-29]. 
Mikami et al. [28] demonstrated a "reciprocal" expression pattern of CK13 and CK17 in oral dysplasia, carcinoma in situ and OSCC. Namely, the appearance of CK17 in carcinoma in situ and OSCC was associated with decreased expression of CK13, which was constantly expressed in the normal and dysplastic squamous epithelia. We found that CK17 was expressed in the suprabasal layer of both cholesteatoma and the meatal skin, whereas basal and parabasal keratinocytes were negative. Interestingly enough, CK17 expression was significantly increased in cholesteatoma tissues compared with the meatal skin $(P<0.001)$. The expression was more pronounced in the invasive group. Moreover, CK17 activation was positively correlated with the number of eroded bones. In contrast, CK13 showed no significant difference and similar expression pattern between cholesteatoma and the skin. To get it more clearly, we conducted a paired comparison between CK13 and CK17 in both skin and cholesteatoma. No significant difference was found in the control skin tissue ( $P=0.313)$, while in cholesteatomas, CK17 expression was significantly increased $(P<0.001)$. This finding could clarify the favoring role of CK17 in acquired cholesteatoma.

We should emphasize a positive role of CK17 in pathogenesis as well as in bone destruction in cholesteatoma. Although cholesteatoma is a nonneoplastic lesion, it attains many characters similar to neoplasms like invasion, migration, proliferation, and recidivation [30]. We suggest that CK17, a potential marker for oral malignancy, can be a marker to predict cholesteatoma aggressiveness. However, this novel finding must be warranted by further investigation with a larger sample-size and also by adding genetic studies which are considered to be limitations in our study. We did not find positive correlation between Ki-67 and CK17 even though both of them were positively correlated with the number of eroded bones. This may indicate that both can be independent markers for the invasiveness of the lesion. In addition, we did not find significant correlation between the age of patients at the time of surgery and the grading score for bone erosion $(r=0.338, P=0.158)$ and this is matching with previous studies [6,7,24]. Mallet et al. [7] did not find significant correlation between age and bone destruction in a larger cohort than our study (91 patients). Regarding the duration of illness, it was difficult to accurately determine it in many of our patients as they gave vague history especially for the onset of symptoms. So, deficient data about this point made it hard to clarify the effect of disease duration on the degree of bone destruction.

In conclusion, we suggested a newly discovered positive role for CK17 in the pathogenesis of acquired cholesteatoma and its ability to destroy bony structures as well. Second, we added supporting evidence that Ki-67 could be considered as a reliable proliferative marker and predictor for cholesteatoma aggressiveness.

\section{CONFLICT OF INTEREST}

No potential conflict of interest relevant to this article was reported.

\section{ACKNOWLEDGMENTS}

We would like to express our sincere gratitude to the Society for Promotion of International Otorhinolaryngology (Tokyo, Japan) organization for partially granting the present work. In addition, many thanks to Professor Shingo Murakami (Otolaryngology Department, Nagoya City University) and Dr Hayato Misawa (Otolaryngology Department, Nagoya Medical Center) for continuous help and advice during this research work.

\section{REFERENCES}

1. Olszewska E, Wagner M, Bernal-Sprekelsen M, Ebmeyer J, Dazert S, Hildmann H, et al. Etiopathogenesis of cholesteatoma. Eur Arch Otorhinolaryngol. 2004 Jan;261(1):6-24.

2. Bujia J, Schilling V, Holly A, Stammberger M, Kastenbauer E. Hyperproliferation-associated keratin expression in human middle ear cholesteatoma. Acta Otolaryngol. 1993 May;113(3):364-8.

3. Juhn SK, Jung MK, Hoffman MD, Drew BR, Preciado DA, Sausen NJ, et al. The role of inflammatory mediators in the pathogenesis of otitis media and sequelae. Clin Exp Otorhinolaryngol. 2008 Sep;1 (3):117-38.

4. Peek FA, Huisman MA, Berckmans RJ, Sturk A, Van Loon J, Grote JJ. Lipopolysaccharide concentration and bone resorption in cholesteatoma. Otol Neurotol. 2003 Sep;24(5):709-13.

5. Olszewska E, Jakimowicz-Rudy J, Knas M, Chilimoniuk M, Pietruski JK, Sieskiewicz A. Cholesteatoma-associated pathogenicity: potential role of lysosomal exoglycosidases. Otol Neurotol. 2012 Jun;33 (4):596-603.

6. Sikka K, Sharma SC, Thakar A, Dattagupta S. Evaluation of epithelial proliferation in paediatric and adult cholesteatomas using the Ki67 proliferation marker. J Laryngol Otol. 2012 May;126(5):460-3.

7. MalletY, Nouwen J, Lecomte-Houcke M, Desaulty A. Aggressiveness and quantification of epithelial proliferation of middle ear cholesteatoma by MIB1. Laryngoscope. 2003 Feb;113(2):328-31.

8. Banco B, Grieco V, Di Giancamillo M, GreciV, Travetti O, Martino P, et al. Canine aural cholesteatoma: a histological and immunohistochemical study. Vet J. 2014 Jun;200(3):440-5.

9. Shiwa M, Kojima H, Moriyama H. Expression of transforming growth factor-alpha (TGF-alpha) in cholesteatoma. J Laryngol Otol. 1998 Aug;112(8):750-4.

10. Yamamoto-Fukuda T, Takahashi H, Terakado M, Hishikawa Y, Koji T. Expression of keratinocyte growth factor and its receptor in noncholesteatomatous and cholesteatomatous chronic otitis media. Otol Neurotol. 2010 Jul;31(5):745-51.

11. Macias MP, Gerkin RD, Macias JD. Increased amphiregulin expression as a biomarker of cholesteatoma activity. Laryngoscope. 2010 Nov;120(11):2258-63.

12. Lee SH, Jang YH, Tae K, Park YW, Kang MJ, Kim KR, et al.Telomerase activity and cell proliferation index in cholesteatoma. Acta Otolaryngol. 2005 Jul;125(7):707-12.

13. Yetiser S, Satar B, Aydin N. Expression of epidermal growth factor, 
tumor necrosis factor-alpha, and interleukin-1alpha in chronic otitis media with or without cholesteatoma. Otol Neurotol. 2002 Sep;23 (5):647-52.

14. Juhasz A, Sziklai I, Rakosy Z, Ecsedi S, Adany R, Balazs M. Elevated level of tenascin and matrix metalloproteinase 9 correlates with the bone destruction capacity of cholesteatomas. Otol Neurotol. 2009 Jun;30(4):559-65.

15. Oger M,Alpay HC, Orhan I, Onalan EE, Yanilmaz M, Sapmaz E. The effect of BMP-2, BMP-4 and BMP-6 on bone destruction of cholesteatoma presence. Am J Otolaryngol. 2013 Nov-Dec;34(6):652-7.

16. Kuo CL. Etiopathogenesis of acquired cholesteatoma: prominent theories and recent advances in biomolecular research. Laryngoscope. 2015 Jan;125(1):234-40.

17. Vennix PP, KuijpersW, Peters TA, Tonnaer EL, Ramaekers FC. Epidermal differentiation in the human external auditory meatus. Laryngoscope. 1996 Apr;106(4):470-5.

18. Vennix PP, Kuijpers W, Peters TA, Tonnaer EL, Ramaekers FC. Keratinocyte differentiation in acquired cholesteatoma and perforated tympanic membranes. Arch Otolaryngol Head Neck Surg. 1996 Aug;122(8):825-32.

19. Sudhoff H, Bujia J, Fisseler-Eckhoff A, Holly A, Schulz-Flake C, Hildmann H. Expression of a cell-cycle-associated nuclear antigen (MIB 1) in cholesteatoma and auditory meatal skin. Laryngoscope. 1995 Nov; 105(11):1227-31.

20. Raynov AM, Moon SK, Choung YH, Hong SP, Park K. Nucleoplasm staining patterns and cell cycle-associated expression of Ki-67 in middle ear cholesteatoma. Am J Otolaryngol. 2005 Sep-Oct;26(5): 296-301.

21. Akdogan V, Yilmaz I, Canpolat T, Ozluoglu LN. Role of Langerhans cells, Ki-67 protein and apoptosis in acquired cholesteatoma: prospective clinical study. J Laryngol Otol. 2013 Mar;127(3):252-9.
22. Chung JH, Lee SH, Park CW, Kim KR, Tae K, Kang SH, et al. Expression of apoptotic vs antiapoptotic proteins in middle ear cholesteatoma. Otolaryngol Head Neck Surg. 2015 Dec;153(6):1024-30.

23. Kuczkowski J, Pawelczyk T, Bakowska A, Narozny W, Mikaszewski B. Expression patterns of Ki-67 and telomerase activity in middle ear cholesteatoma. Otol Neurotol. 2007 Feb;28(2):204-7.

24. Aslier M, Erdag TK, Sarioglu S, Guneri EA, Ikiz AO, Uzun E, et al. Analysis of histopathological aspects and bone destruction characteristics in acquired middle ear cholesteatoma of pediatric and adult patients. Int J Pediatr Otorhinolaryngol. 2016 Mar;82:73-7.

25. Kim HJ, Tinling SP, Chole RA. Increased proliferation and migration of epithelium in advancing experimental cholesteatomas. Otol Neurotol. 2002 Nov;23(6):840-4.

26. Lee RJ, Sidey C, Narula AA, James RF. The nature of the epithelium in acquired cholesteatoma: part 3: cytokeratin patterns in aural epithelial and cholesteatoma cells grown in cell culture. Clin Otolaryngol Allied Sci. 1994 Dec;19(6):516-20.

27. Toyoshima T, Vairaktaris E, Nkenke E, Schlegel KA, Neukam FW, Ries J. Cytokeratin 17 mRNA expression has potential for diagnostic marker of oral squamous cell carcinoma. J Cancer Res Clin Oncol. 2008 Apr;134(4):515-21.

28. MikamiT, Cheng J, Maruyama S, KobayashiT, Funayama A, Yamazaki M, et al. Emergence of keratin 17 vs. loss of keratin 13: their reciprocal immunohistochemical profiles in oral carcinoma in situ. Oral Oncol. 2011 Jun;47(6):497-503.

29. Jin L, Wang G. Keratin 17: a critical player in the pathogenesis of psoriasis. Med Res Rev. 2014 Mar;34(2):438-54.

30. Desloge RB, Carew JF, Finstad CL, Steiner MG, Sassoon J, Levenson MJ, et al. DNA analysis of human cholesteatomas. Am J Otol. 1997 Mar;18(2):155-9. 